The application of molecular genetics to muscle, the nervous system and cell lineage is the hallmark of research on C. elegans, as is made evident in several cogent chapters. For example, the ability easily to identify and propagate paralysed mutants defective in a myosin heavy chain made possible the first complete sequence of this polypeptide. Identification of over 100 genes controlling various aspects of development relied on many of the characteristics apparent to Brenner in his choice of the worm, especially its small number of cells and short generation time. Yet although the excitement of recent work on sex determination and germline development is amply conveyed, a chapter devoted to the cuticle and molecular genetics of collagen would have been welcome - several genes defined by morphological and behavioural mutants encode collagens.

The final third of the book is taken up with helpful appendices. The summary of methods is not a complete laboratory manual, but is a good place to start. A 60page gene list includes invaluable information for the novice (or expert), such as how easy it is to score the phenotypes of the various mutants.

For any biologist, this monograph can serve as an enticement to study a favourite problem in the worm, or as a source of unanswered questions. One hopes that those so encouraged will retain the cooperative spirit, the high standards and the love of this organism that have characterized the field over the past two decades.

PaulW. Sternberg is in the Division of Biology, California Institute of Technology, Pasadena, California 91125, USA.

\section{The number of the rose}

\section{John D. Barrow}

Between Quantum and Cosmos: Studies and Essays in Honor of John Archibald Wheeler. Edited by W.H. Zurek, A. van der Merwe and W.A. Miller. Princeton University Press:1988. Pp.623. \$49.50.

"TIME is God's way of keeping things from happening all at once." Anyone who treasures this amongst their favourite aphorisms is clearly a little out of the ordinary.

John Wheeler is certainly such a person. In honour of his seventy-fifth birthday many of his former students and collaborators cornered six issues of the monthly journal Foundations of Physics and filled them with research papers and reviews on subjects to which Wheeler has made fundamental contributions or in which he has a strong interest. Here, these articles are gathered up into a single volume. The result is different both in style and intent from the earlier Wheeler Festschrift, Magic without Magic, published a decade ago. There are many more contributions, at a much higher technical level, and there are no general biographical articles about Wheeler's scientific career and style of working. Instead there are annotated highlights and characteristic artwork from his most important papers. Yet despite these adornments and the catchy titles of many contributions, this is not a popular book nor even one for scientific outsiders.

The articles fall into five principal categories. The first spans those areas of nuclear physics with a wheelerian pedigree; in particular, the development of the theory of fission (with Niels Bohr), the S-matrix, nuclear models and the role tive measure - the shortest program able to generate the sequence - because account must be taken of the difficulty of arriving at the minimal program. Some proposals for achieving this find their way into Bennett's contribution, although the best ideas, which concentrate upon the amount of entropy generated by the program of minimum length, were made after his article was written.

Finally, one should highlight the article by Geroch and Hartle, which describes what it means for a measurable physical quantity to be computable in the sense of Turing, Church and Post. In our mundane experience of physics, non-computable functions have not emerged naturally. It is, though, quite possible for the varieties of differential equation familiar in physics - ordinary differential equations or hyperbolic wave equations - to possess solutions which are non-computable functions of well-posed initial conditions, if one allows the initial behaviour to be not entirely smooth but contain creases and cusps like those found at the point of a cone. Although it is usual for physicists to discount such kinky starting conditions as physically unrealistic, it is not clear that they should do so given the noncontinuum nature of classical physics.

Geroch and Hartle discuss a recently discovered example, which arises in quantum cosmology. Here, an observable is found to be equal to a sum of quantities, which must be evaluated for all the compact four-geometries. But the infinite list of these geometries is known to be non-computable. This does not necessarily mean that the observable cannot be computed and predicted - there might be another way that avoids listing the unlistable and so is computable. Nonetheless, it provides an interesting example of how difficult it may prove to answer some of the questions posed in cosmology and particle physics. The ultimate capability of the human brain and of all our computational machines may fall short of that required to rationalize things.

The book makes interesting reading, but it is disappointing in some respects. The articles were originally intended for a journal, and the authors might have written more generally, more speculatively and in a style suited for a wider audience had they set out to compile a book. Aside from the cache of contributions on computation, they show primarily what can be achieved by technical expertise rather than creative imagination. Because John Archibald Wheeler has displayed, and continues to display, the power of the imagination more powerfully than almost any other physicist, that is a pity. But perhaps it shows simply that only John Wheeler can write the articles that John Wheeler writes.

John D. Barrow is in the Astronomy Centre, University of Sussex, Brighton BN1 9QH, UK. 